\title{
EXPERIMENTOS SIMPLES USANDO FOTOMETRIA DE CHAMA PARA ENSINO DE PRINCÍPIOS DE ESPECTROMETRIA ATÔMICA EM CURSOS DE QUÍMICA ANALÍTICA
}

\author{
Fabiano Okumura e Éder T. G. Cavalheiro* \\ Instituto de Química de São Carlos, Universidade de São Paulo, CP 780, 400, 13560-970 São Carlos - SP \\ Joaquim A. Nóbrega \\ Departamento de Química, Universidade Federal de São Carlos, CP 676, 13560-970 São Carlos - SP
}

Recebido em 19/5/03; aceito em 30/1/04; publicado na web em 17/6/04

\begin{abstract}
SIMPLE FLAME PHOTOMETRIC EXPERIMENTS TO TEACH PRINCIPLES OF ATOMIC SPECTROMETRY IN UNDERGRADUATE ANALYTICAL CHEMISTRY COURSES. The purpose of this paper is the development of simple strategies to teach basic concepts of atomic spectrometry. Metals present in samples found in the daily lives of students are determined by flame atomic emission spectrometry (FAES). FAES is an accurate, precise, and inexpensive analytical method often used for determining sodium, potassium, lithium, and calcium. Historical aspects and their contextualization for students are also presented and experiments with samples that do not require pre-treatment are described.
\end{abstract}

Keywords: flame atomic emission photometry; education in analytical chemistry; instrumental analysis.

\section{INTRODUÇÃO}

A fotometria de chama é a mais simples das técnicas analíticas baseadas em espectroscopia atômica. Nesse caso, a amostra contendo cátions metálicos é inserida em uma chama e analisada pela quantidade de radiação emitida pelas espécies atômicas ou iônicas excitadas. Os elementos, ao receberem energia de uma chama, geram espécies excitadas que, ao retornarem para o estado fundamental, liberam parte da energia recebida na forma de radiação, em comprimentos de onda característicos para cada elemento químico ${ }^{1-4}$.

Apesar da simplicidade da técnica, diversos conceitos importantes estão envolvidos no desenvolvimento de experimentos usando a fotometria de chama, desde os princípios de espectroscopia até a estatística no tratamento de dados, passando por preparo de amostra e eliminação de interferências ${ }^{1,2}$.

Este trabalho é parte de um projeto que objetiva o ensino de princípios de métodos óticos de análise para ensino superior e escolas técnicas, utilizando a fotometria de chama para análise de amostras do cotidiano dos alunos. Considerando o baixo custo do instrumento usado em relação à sua ampla aplicabilidade, a técnica oferece muitas opções didáticas. $\mathrm{O}$ uso de amostras presentes no cotidiano é reconhecidamente importante para atrair a atenção do estudante, melhorando o aproveitamento do conteúdo abordado. Aspectos como a interdisciplinaridade e o cotidiano são incentivados pelas novas propostas de ensino no País, tais como a Lei das Diretrizes e Bases da Educação, além dos Parâmetros Curriculares Nacionais $(\mathrm{PCN})$, que foram propostos para o ensino fundamental e médio, com o intuito de melhorar a participação dos alunos nas aulas e despertar o seu interesse para o conteúdo abordado ${ }^{5}$, mas podem ser úteis também no ensino superior. Essas propostas são plenamente contempladas nos experimentos propostos.

A introdução de um contexto histórico também contribui positivamente na proposta de atrair a atenção dos alunos. Uma breve discussão sobre o desenvolvimento dos métodos de espectroscopia atômica é apresentada a seguir. Segundo propostas recentes, o contexto

*e-mail: cavalheiro@iqsc.usp.br histórico deve ser apresentado aos alunos, de maneira a contribuir no processo de ensino, apresentando-lhes o desenvolvimento científico, seus erros e discussões ${ }^{5}$. Isso possibilita aos estudantes a vivência do processo de criação científica e o desenvolvimento da percepção de que o mesmo, tal como as demais obras humanas, é marcado por uma evolução gradual decorrente do trabalho de equipes multi-disciplinares e que as abordagens são freqüentemente revistas ou aprofundadas, em função da disponibilidade de novos dados experimentais ou mesmo da substituição de paradigmas.

Nos experimentos aqui propostos, as amostras analisadas foram soro fisiológico, águas minerais, bebidas isotônicas e medicamentos anti-depressivos à base de lítio. A natureza dessas amostras sugere seu aproveitamento também em cursos de farmácia. Algumas das práticas foram aplicadas em aulas e os resultados, avaliados em provas e questionários.

\section{Considerações sobre o desenvolvimento da espectroscopia atômica $^{2,6,7}$}

A apresentação da evolução histórica sobre o conteúdo abordado é recomendada pelas razões já citadas. No caso da espectroscopia atômica, devido à sua grande relevância para o avanço das ciências naturais, há vários textos relacionados com o tema, destacando-se os de Jarrell ${ }^{6}$, que apresenta um histórico da análise por emissão atômica entre 1660-1950, o livro de Lajunen ${ }^{2}$ sobre análise espectroquímica por emissão e absorção atômicas e o artigo de Filgueiras ${ }^{7}$ sobre as relações da espectroscopia e a química, entre inúmeros outros. Os parágrafos abaixo são um resumo do que citam estes autores ${ }^{2,6,7}$.

Tais textos destacaram a teoria corpuscular da luz de Isaac Newton (1666), que foi o primeiro a observar a decomposição da luz branca ao incidir em um prisma, resultando no aparecimento de diferentes cores, o que o levou a supor que a luz seria composta de partículas minúsculas, que se deslocariam em altas velocidades.

Já Christian Huygens, físico holandês, apresentou em 1678 a teoria ondulatória da luz que, atualmente, é mais aceita.

A construção das grades de difração, iniciada em 1786, pelo astrônomo americano David Rittenhouse possibilitou avanços tecnológicos que permitiram a construção de um espectroscópio por 
Joseph Fraunhofer, entre 1814 e 1824. O espectroscópio é um instrumento ótico que possibilita a separação de radiações com distintos comprimentos de onda.

Um dos desenvolvimentos interessantes ao longo do século XIX é a observação de linhas escuras no espectro contínuo da luz solar. Essas linhas escuras, foram observadas por William Hyde Wollaston em 1802, porém só posteriormente foram explicadas como causadas por processos de auto-absorção gerados por nuvem de átomos frios, presentes na periferia solar. Ressalta-se que o termo frio aqui utilizado se justifica comparativamente às temperaturas observadas no núcleo do sol.

Em 1859, Bunsen desenvolveu um queimador no qual se observava a intensidade de emissão dos elementos de forma mais evidente. Kirchhoff reconheceu que as linhas negras do espectro contínuo, descritas por Wollaston e posteriormente também observadas por Fraunhofer, coincidiam com as linhas de emissão de sais introduzidos em uma chama.

Trabalhando juntos Robert Bunsen, químico, e Gustav Kirchhoff, físico, estudaram o espectro de emissão de uma amostra cujas linhas espectrais não correspondiam a nenhum elemento conhecido. Eles o denominaram césio, do grego caesius $=$ azul-celeste, pois o espectro de emissão apresentava linhas azuis. No ano seguinte, um novo elemento foi descoberto, o rubídio, do grego rubidus, pois o seu espectro de emissão continha linhas vermelhas, da cor de um rubi. Esses pesquisadores deram um passo decisivo para a espectroscopia de emissão em chama, ao reconhecer que linhas espectrais emitidas por metais ocorrem em comprimentos de onda definidos, independente dos ânions que estão em solução.

Em 1860, Bunsen e Kirchhoff demonstraram para um grupo de geólogos como identificar elementos como ferro, cobre, chumbo, sódio e potássio em minérios, através da coloração da chama em um queimador especialmente designado e, atualmente, denominado bico de Bunsen. A amostra sólida era dissolvida em água e a solução resultante, introduzida em uma chama. Pode-se estimar as concentrações dos elementos pela comparação da intensidade das cores de soluções de concentrações conhecidas com a intensidade das soluções de amostra sólida de minérios. Posteriormente, construíram um espectroscópio representado pelo esquema da Figura 1. Uma representação mais detalhada do espectroscópio pode ser vista na internet ${ }^{8}$.

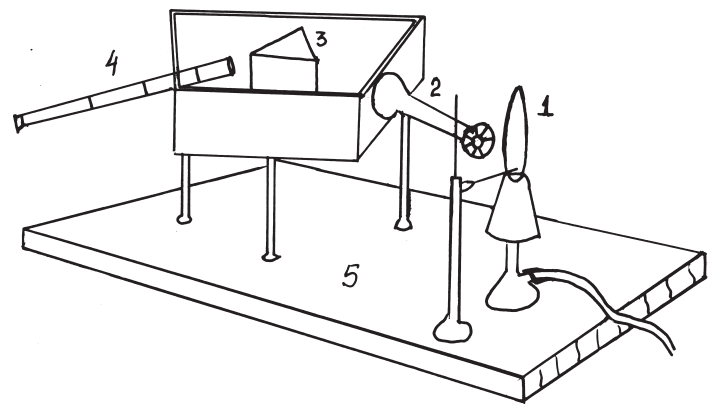

Figura 1. Esquema do espectroscópio construído por Bunsen e Kirchhoff 1- chama, fonte de excitação; 2-colimador; 3-prisma; 4- telescópio e 5plataforma

O espectroscópio de Bunsen-Kirchhoff, representado na Figura 1, é um aparelho usado para efetuar medidas do comprimento de onda de uma radiação luminosa. Seu funcionamento baseia-se na dispersão da luz. É constituído de uma plataforma sobre a qual se colocam um colimador, uma luneta e um projetor, todos apresentados na Figura 1. O colimador é formado por uma fenda discreta, de abertura regulável, colocada no plano focal de uma lente convergen- te, que faz a luz incidir sobre um prisma colocado em um suporte. $\mathrm{O}$ raio que emerge do prisma incide na luneta focada no infinito, de modo a formar uma imagem nítida ${ }^{8}$.

O projetor consiste de uma lente convergente em cujo foco posiciona-se um dispositivo contendo uma escala graduada calibrada, de modo que cada marca corresponda a uma raia monocromática. A escala, devidamente iluminada, projeta uma imagem na mesma direção da luz refratada do prisma permitindo, simultaneamente, a leitura e colimação ${ }^{8}$.

Em 1868 realizaram-se estudos sobre o espectro solar durante os eclipses. Pierre Janssen acoplou uma luneta a um espectroscópio e identificou o hidrogênio como elemento principal. Joseph Norman Lockyer, na mesma época e com o mesmo sistema, analisou o espectro de linhas do sol e identificou um novo elemento, ao qual denominou hélio, em homenagem ao deus grego do sol. Apenas em 1895, o elemento hélio foi identificado na Terra pelo escocês William Ramsay.

A partir daí surgiram duas vertentes voltadas para a análise qualitativa e quantitativa, enquanto a espectroscopia atômica seria também usada no desenvolvimento da teoria atômica. Lajunen ${ }^{2}$ apresentou uma linha cronológica para o desenvolvimento das técnicas baseadas na espectroscopia atômica em química analítica, durante o século XX.

Os desenvolvimentos feitos a partir dessas observações levaram a erros e acertos, podendo ser usados para demonstrar que o desenvolvimento científico se dá por etapas, que nem sempre as conclusões são verdades absolutas e que as teorias científicas são revistas e freqüientemente aprimoradas.

As referências citadas e diversos livros e textos de química geral abordam o tema com profundidade e grande riqueza de detalhes ${ }^{8-11}$. Tais textos podem ser consultados pelos alunos antes dos experimentos, como fonte de pesquisa extra-classe, recomendada pelo professor.

\section{Processos que ocorrem durante a medida por fotometria de chama}

A espectroscopia atômica baseia-se em métodos de análise de elementos de uma amostra, geralmente líquida, que é introduzida em uma chama, na qual ocorrem fenômenos físicos e químicos, como evaporação, vaporização e atomização. Um esquema dos fenômenos que ocorrem na chama é apresentado na Figura 2. Para que todos esses processos possam ocorrer em tempos de residência tipicamente inferiores a 5 min, é necessário que amostras líquidas sejam convertidas em um aerossol líquido-gás com partículas inferiores a 5$10 \mu \mathrm{m}$ para introdução na chama.

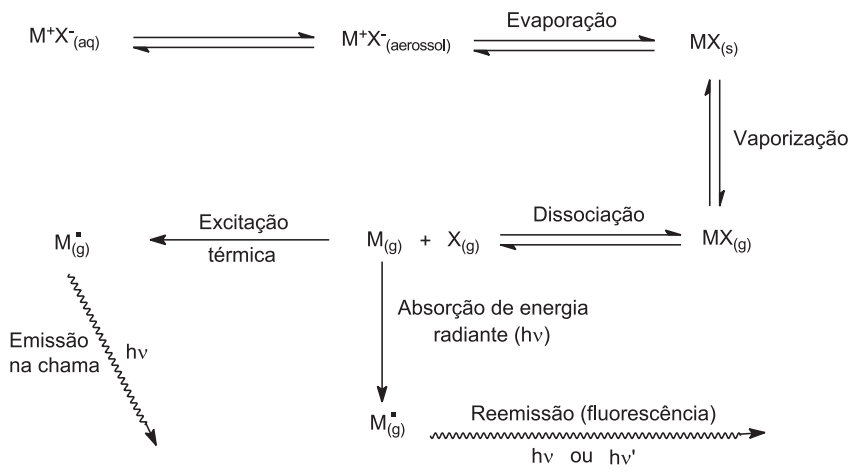

Figura 2. Esquema das reações que ocorrem na chama

A energia eletrônica é quantizada, isto é, apenas certos valores de energia eletrônica são possíveis. Isso significa que os elétrons só podem ocupar certos níveis de energia discretos e que eles absorvem ou emitem energias em quantidades discretas, quando se movem de um orbital para outro. Quando o elétron é promovido do estado fun- 
damental para um estado excitado, ocorre o fenômeno de absorção e quando este retorna para o estado fundamental observa-se o processo de emissão9.

Uma vez que um átomo de um determinado elemento origina um espectro característico de raias, conclui-se que existem diferentes níveis energéticos, e que estes são característicos para cada elemento. Além das transições entre os estados excitados e o fundamental, existem também transições entre os diferentes estados excitados. Assim, um espectro de emissão de um dado elemento pode ser relativamente complexo. Considerando que a razão entre o número de átomos nos estados excitados e o número de átomos no estado fundamental é muito pequena, pode-se considerar que o espectro de absorção de um dado elemento é associado às transições entre o estado fundamental e os estados de energia mais elevados. Desta forma, um espectro de absorção é mais simples que um espectro de emissão ${ }^{3}$.

Átomos na fase gasosa podem ser excitados pela própria chama ou por uma fonte externa. Se forem excitados pela chama, ao retornarem para o estado fundamental, liberam a energia na forma de radiação eletromagnética. Essa é a base da espectrometria de emissão atômica que, antigamente, era conhecida como fotometria de chama e é utilizada largamente em análises clínicas, controle de qualidade de alimentos, além de inúmeras outras aplicações, para averiguar a quantidade de íons de metais alcalinos e alcalino-terrosos, como sódio, potássio, lítio e cálcio ${ }^{1}$.

Esses elementos emitem radiação eletromagnética na região do visível em uma chama ar-gás combustível (GLP), que opera em uma temperatura entre 1700 e $1900{ }^{\circ} \mathrm{C}^{1,4}$. Dessa forma, a energia fornecida é baixa, porém suficiente para excitar $\mathrm{Na}, \mathrm{K}, \mathrm{Li}$ e Ca e, conseqüentemente, gerar a emissão de linhas atômicas características para cada elemento. A intensidade de cada linha emitida depende da concentração da espécie excitada e da probabilidade de ocorrência da transição eletrônica.

Os valores de energia da primeira ionização para $\mathrm{Li}, \mathrm{Na}, \mathrm{K}$ e Ca são, respectivamente ${ }^{9}, 520,497,419$ e 590 kJ mol de átomos ${ }^{-1}$, enquanto os valores de energia de excitação são, respectivamente ${ }^{10}$, 173, 203, 154 e $280 \mathrm{~kJ}$ mol de átomos ${ }^{-1}$.

Considerando a magnitude destas energias, o professor pode explorar a existência de possíveis interferências, considerando o grau de adiantamento da turma. Na prática, misturas dos íons poderiam ser analisadas.

As energias da segunda ionização são muito elevadas tornandose inviáveis na temperatura da chama ar-GLP usada. Por exemplo, para a formação de $\mathrm{Li}^{2+}$ a partir de $\mathrm{Li}^{+}$, tal energia ${ }^{9}$ é de $7298 \mathrm{~kJ}$ mol de ions ${ }^{-1}$.

\section{PARTE EXPERIMENTAL}

\section{Equipamentos}

Nos experimentos realizados foram utilizados os seguintes equipamentos: fotômetro de chama DM-61 (Digimed) com câmara de nebulização para introdução de combustível e ar comprimido, banho de ultra-som USC-1400 (Unique) e micropipetadores Eppendorf com diferentes volumes. Podem ser usados pipetadores de volumes fixos entre 10 e $1000 \mu \mathrm{L}$ ou pipetadores de volumes variáveis de 10 - 100 e $100-1000 \mu \mathrm{L}$.

\section{Reagentes e soluções}

Em todos os experimentos foram utilizadas soluções estoque em concentração $1000,0 \mathrm{mg} \mathrm{L}^{-1}$ ( $\mu \mathrm{g} \mathrm{mL}^{-1}$, ppm, partes por milhão), preparadas a partir de cloretos de lítio, sódio, potássio e carbonato de cálcio (Mallinckrodt). As soluções foram preparadas com água desionizada, imediatamente após secagem dos sais por $24 \mathrm{~h}$, a $110^{\circ} \mathrm{C}^{3}$. O carbonato de cálcio foi dissolvido com $\mathrm{HCl}$ concentrado e a solução resultante foi diluída para 1 L com água desionizada.

Também foi preparada uma solução estoque de cloreto de lantânio (Aldrich) $10 \% \mathrm{~m} \mathrm{v}^{-1}$ em lantânio, usada para as determinações de cálcio, nas quais a concentração de trabalho foi ajustada por diluição do estoque para $1 \% \mathrm{~m} \mathrm{v}^{-1}$ em lantânio.

\section{Procedimentos experimentais}

Experimento I - Determinação de cloreto de sódio em soro fisiológico

Este experimento é fácil de ser conduzido e apresenta um apelo interessante, considerando-se a aplicação médica e a importância do soro fisiológico na medicina, o que atrai de maneira positiva a atenção dos alunos para a prática.

O soro fisiológico é a solução universalmente adotada para prover o organismo de sódio e cloro, assim chamado porque é isotônico em relação à fase líquida do corpo. É uma solução de sais em água em uma concentração isotônica, ou seja, de concentração igual à das células. O sódio e o cloro são absorvidos juntamente com a água, devido ao déficit extracelular desses íons ${ }^{12}$.

Pode ser uma alternativa interessante para alunos de cursos de farmácia ou mesmo de áreas como engenharia química e outros, podendo ser realizado em uma aula com duração de 4 h, desde que não seja feita uma introdução teórica longa da técnica. Neste caso, há também a vantagem de reagentes e insumos de custo relativamente baixo.

Nesse experimento, os alunos convertem a concentração do soro 0,900\% em NaCl, para a concentração de sódio $3540 \mathrm{mg} \mathrm{L}^{-1} \mathrm{em} \mathrm{Na}^{+} \mathrm{e}$ solicita-se que estabeleçam uma curva analítica para determinação do teor de sódio. Uma sugestão é que o soro deve ser diluído 100 vezes com água desionizada e uma curva analítica entre 0 e $50 \mathrm{mg} \mathrm{L}^{-1} \mathrm{de}$ sódio deve ser utilizada, neste caso. Usando amostras menos concentradas, outras regiões lineares podem ser utilizadas.

Um aspecto importante neste experimento consiste em determinar o teor de cloreto empregando uma titulação argentométrica, utilizando nitrato de prata como titulante e cromato de sódio como indicador (método de $\mathrm{Mohr}^{3}$ ) e, em seguida, comparar os resultados obtidos, como na Tabela 1.

A curva analítica obedeceu à relação abaixo:

$\mathrm{Y}=2,46+0,95\left[\mathrm{Na}^{+}\right](\mathrm{r}=0,9979, \mathrm{n}=5)$

O experimento foi aplicado em um curso de análise instrumental e foram obtidos resultados adequados, tendo um aproveitamento

Tabela 1. Teores de $\mathrm{NaCl}$ em soro fisiológico obtidos por fotometria e argentometria, pela equipe responsável pelo curso

\begin{tabular}{cccccc}
\hline Rotulado $(\%)$ & Fotometria* $(\%)$ & Argentometria** $(\%)$ & $\mathrm{E}_{1}(\%)$ & $\mathrm{E}_{2}(\%)$ & $\mathrm{E}_{3}(\%)$ \\
\hline 0,90 & $0,89 \pm 0,01$ & $0,94 \pm 0,01$ & 1,12 & 4,25 & 5,32 \\
\hline
\end{tabular}

* média de 5 determinações; ** média de 3 determinações; $\mathrm{E}_{1}$ - erro relativo entre o valor rotulado e o valor encontrado; $\mathrm{E}_{2}$ - erro relativo entre o valor rotulado e o método comparativo e $\mathrm{E}_{3}$ - erro relativo entre o valor encontrado e o método comparativo. 
satisfatório da classe, representado por acertos através de relatórios e questões formuladas em prova e em sala de aula. Exemplo dos resultados obtidos pelos alunos e o tratamento dos dados é apresentado na Tabela 2, na qual se pode observar uma grande variação nos resultados. Uma discussão bastante ampla pode ser feita a respeito destas diferenças, evidenciando suas causas e como melhorar os resultados em alguns casos.

Tabela 2. Resultados obtidos pelos alunos, em aula, para o teor de cloreto de sódio em uma amostra de soro fisiológico

\begin{tabular}{ccc}
\hline Turma & $\mathrm{NaCl} / \%$ & $\mathrm{E}^{*} / \%$ \\
\hline 1 & 0,96 & 6,67 \\
2 & 0,83 & $-7,78$ \\
3 & 0,98 & 8,89 \\
4 & 0,98 & 8,89 \\
5 & 0,84 & $-6,67$ \\
6 & 0,92 & 2,22 \\
7 & 0,91 & 1,11 \\
8 & 0,97 & 7,78 \\
9 & 0,93 & 3,33 \\
\hline
\end{tabular}

Média da turma: 0,93 ( $\mathrm{n}=9)$; Desvio padrão: $\pm 0,06$ e $\mathrm{n}^{\circ} \mathrm{de}$ determinações: $\mathrm{n}=9$

* Erro relativo ao valor rotulado de $0,90 \%$

O uso da amostra de soro mostrou-se um ponto interessante para despertar o interesse dos alunos, conforme suas respostas a um questionário aplicado durante o curso. Neste caso, vinte e um alunos participaram da aula prática. $\mathrm{O}$ uso da amostra do cotidiano atendeu às expectativas de atrair atenção para o problema abordado e, conseqüentemente, para o conteúdo relativo à técnica envolvida, além da aquisição e tratamento de dados.

A maioria, 78\%, considerou a estratégia adotada interessante e produtiva no aspecto de chamar sua atenção para o problema envolvido e para o conteúdo abordado, de acordo com suas respostas ao questionário.

\section{Experimento II - Determinação de lítio em comprimidos anti- depressivos}

O lítio na forma de carbonato pode ser encontrado em farmácias, como medicamento anti-depressivo e para o tratamento de outras doenças que envolvem o sistema nervoso central ${ }^{13}$. Neste experimento, a amostra de lítio estava na forma de drágeas, que podem conter 300 ou $450 \mathrm{mg}$ de carbonato de lítio. O comprimido foi triturado em almofariz, dissolvido em ácido clorídrico $0,1 \mathrm{~mol} \mathrm{~L}^{-1} \mathrm{e}$, em seguida, transferido para um balão volumétrico de $250 \mathrm{~mL}$ e o volume completado com água bidestilada. Uma alíquota desta solução foi diluída $10 \mathrm{x}$, para que a concentração de lítio ficasse no intervalo linear. A solução resultante foi colocada em um banho de ultra-som por
10 min. A curva analítica foi obtida entre 0 e $30 \mathrm{mg} \mathrm{L}^{-1}$ e foram realizadas as leituras das soluções de referência e da amostra. Os resultados obtidos para a média de cinco determinações mostraram um erro relativo de $-0,60 \%$ entre o valor encontrado $\left(298,20 \pm 0,53 \mathrm{mg} \mathrm{L}^{-1}\right)$ e o rotulado $\left(300,00 \mathrm{mg} \mathrm{L}^{-1}\right)$

A curva analítica obedeceu à relação:

$\mathrm{Y}=-1,86+1,11\left[\mathrm{Li}^{+}\right](\mathrm{r}=0,9961, \mathrm{n}=5)$

No presente trabalho, os comprimidos foram doados pelo fabricante, já que se trata de um medicamento com venda controlada. Os alunos devem ser alertados para tomar cuidado com o manuseio, evitando ingestão da amostra.

O lítio também vem sendo usado na preparação de baterias. Sua determinação neste tipo de amostras poderia despertar grande interesse quanto ao aspecto ambiental.

\section{Experimento III - Determinação de cálcio em águas naturais}

Neste caso, os teores de cálcio foram determinados em alíquotas de água mineral de diferentes marcas.

$\mathrm{Na}$ determinação de cálcio surge um desafio interessante a ser vencido pelos alunos, pois pode ocorrer interferência negativa causada por fosfatos e silicatos presentes na água ${ }^{1}$, o que provoca diminuição significativa do sinal. Eventualmente, óxido de cálcio pode se formar na chama, conforme esquema da Figura 2. Uma das formas de contornar o problema é a adição de lantânio em meio ácido, que reage com os interferentes liberando o $\mathrm{Ca}^{2+}$, que pode então ser determinado. Os resultados obtidos na determinação do $\mathrm{Ca}^{2+}$ nas águas minerais são apresentados na Tabela 3. A literatura também sugere o uso de EDTA para contornar essas interferências ${ }^{1,3}$. Quando as medidas são feitas sem o emprego do lantânio, ocorre forte supressão do sinal, como pode ser visto na Tabela 3 .

A curva analítica obedeceu a Lei de Beer no intervalo de 0 a $30 \mathrm{mg} \mathrm{L}^{-1}$ e pode ser representada pela seguinte equação:

$\mathrm{Y}=1,29+0,88\left[\mathrm{Ca}^{2+}\right](\mathrm{r}=0,9985, \mathrm{n}=5)$

\section{Experimento IV - Determinação de $\mathrm{Na}^{+}$e $\mathrm{K}^{+}$em bebidas} isotônicas

Neste experimento, os teores de $\mathrm{Na}^{+}$e $\mathrm{K}^{+}$foram determinados em amostras de bebidas isotônicas. A determinação desses elementos químicos é relevante e, em alguns países, a sua declaração no rótulo de alimentos é obrigatória, devido à influência que ambos exercem sobre o controle da pressão arterial. Pessoas com tendência à hipertensão devem privilegiar alimentos com maior teor de $\mathrm{K}^{+} \mathrm{e}$ menor teor de $\mathrm{Na}^{+}$

As amostras foram diluídas 1:100 $\mathrm{v} \mathrm{v}^{-1}$ em água destilada. Apesar da presença de corantes na amostra original, a solução resultante apresentou-se homogênea e incolor.

Os resultados obtidos na determinação do $\mathrm{Na}^{+}$e $\mathrm{K}^{+}$nas bebidas são apresentados na Tabela 4.

Tabela 3. Teores de cálcio em águas naturais

\begin{tabular}{ccccc}
\hline Amostra & Rotulado $\left(\mathrm{mg} \mathrm{L}^{-1}\right)$ & \multicolumn{2}{c}{ Encontrado* $\left(\mathrm{mg} \mathrm{L}^{-1}\right)$} & \multicolumn{2}{c}{ Erro Relativo (\%) } \\
& \multicolumn{3}{c}{ Ausência de La } & Presença de La \\
\hline 1 & 60,12 & $15,81 \pm 0,41$ & $58,97 \pm 0,86$ & $-1,95$ \\
2 & 16,40 & - & $17,61 \pm 0,21$ & 6,87 \\
3 & 17,09 & - & $17,83 \pm 0,21$ & 4,33 \\
4 & 26,85 & $13,57 \pm 0,41$ & $26,80 \pm 0,23$ & $-0,18$ \\
\hline
\end{tabular}

* média de 3 determinações; - sem sinal 
Tabela 4. Teores de sódio e potássio em isotônicos

\begin{tabular}{cccc}
\hline Elemento & $\begin{array}{c}\text { Rotulado } \\
\left(\mathrm{mg} \mathrm{L}^{-1}\right)\end{array}$ & $\begin{array}{c}\text { Encontrado* } \\
\left(\mathrm{mg} \mathrm{L}^{-1}\right)\end{array}$ & $\begin{array}{c}\text { Erro Relativo } \\
(\%)\end{array}$ \\
\hline $\mathrm{Na}$ & 90,0 & $86,3 \pm 0,1$ & $-4,11$ \\
$\mathrm{~K}$ & 240,0 & $241,7 \pm 1,1$ & 0,70 \\
\hline
\end{tabular}

* média de 5 determinações.

As curvas analíticas obedeceram a Lei de Beer no intervalo em estudo. Neste caso, os intervalos obtidos na diluição $1: 100 \mathrm{v} \mathrm{v}^{-1}$ foram de 0 - 50 para $\mathrm{Na}^{+}$e $0-30$ ppm para $\mathrm{K}^{+}$, regidos pelas seguintes equações:

$\mathrm{Y}=2,46+0,95\left[\mathrm{Na}^{+}\right](\mathrm{r}=0,9979, \mathrm{n}=5)$

$\mathrm{Y}=0,84+0,94\left[\mathrm{~K}^{+}\right](\mathrm{r}=0,9978, \mathrm{n}=5)$

O intervalo de concentrações de trabalho pode ser definido por cálculos realizados pelos alunos, considerando-se os valores rotulados e a sensibilidade do equipamento. Caso o valor seja desconhecido, os intervalos deverão ser definidos por tentativa e erro.

\section{CONCLUSÃO}

O desenvolvimento destes experimentos com base em amostras de uso cotidiano exerceu forte atração em relação à atenção dos alunos para o conteúdo abordado.

A espectrometria de emissão atômica por chama (fotometria de chama) é uma alternativa instrumental de baixo custo para determinação de $\mathrm{Li}^{+}, \mathrm{Na}^{+}, \mathrm{K}^{+}$e $\mathrm{Ca}^{2+}$ em diferentes amostras simples e que requerem tratamento prévio mínimo. Isso pode ser útil para cursos com tempos de aula reduzidos ou escolas com laboratórios didáticos sem recursos instrumentais sofisticados, como espectrômetros de absorção atômica, para introdução à análise instrumental. Também pode representar uma alternativa interessante para alunos de cursos como farmácia e outras áreas, dado o apelo exercido pelas amostras.

Aspectos importantes como o tratamento de dados e estatística de resultados também podem ser abordados, usando tais experimentos.

\section{AGRADECIMENTOS}

Os autores agradecem à FAPESP pelo auxílio financeiro (proc. 00/14486-2) e a bolsa concedida a F. Okumura (processo 02/05645-5).

\section{REFERÊNCIAS}

1. Dean, J. A.; Flame Photometry, McGraw-Hill: New York, 1960.

2. Lajunen, L. H. J.; Spectrochemical Analysis by Atomic Absorption and Emission, Royal Society of Chemistry: Cambridge, 1992.

3. Jeffrey, H.; Bassett, J.; Mendham, J.; Denney, R. C.; Vogel: Análise Química

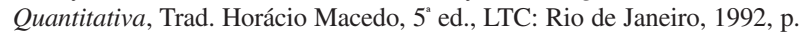
629

4. Skoog, D. A.; West, D. M.; Holler, F. J.; Analytical Chemistry: An Introduction, $6^{\mathrm{a}}$ ed., Saunders: Chicago, 1994

5. Ministério da Educação, Parâmetros Curriculares Nacionais, Governo Federal, MEC/SEMTEC, Brasília, 2002

6. Jarrell, R. F.; J. Chem. Educ. 2000, 77, 573

7. Filgueiras, C. A. L.; Química Nova na Escola 1996, nㅡ 3, 22.

8. http://www.ct.infn.it/ museo/KIRCHO.HTM, acessada em Setembro 2003.

9. Whitten, K. W.; Davis, R. E.; Peck, M. L.; General Chemistry with Qualitative Analysis, 5 ${ }^{\text {th }}$ ed., Saunders: New York, 1996.

10. Harrison, G. R.; Massachussetts Institute of Technology Wavelength Tables, The M. I. T. Press, $2^{\text {nd }}$ ed., 1969, USA, p. 429.

11. Atkins, P.; Jones, L. Em Princípios de Química; trad. Caracelli, I.; Zukerman-Schpector, J., coord., Bookman: Porto Alegre, 2001.

12. www.geocities.com/CollegePark/Residence/4589/aguasoro.htm, acessada em Abril 2003.

13. Fuchs, F. D.; Wannmacher, L.; Farmacologia Clínica: Fundamentos da Terapia Racional, 2a ed., Guanabara Koogan: Rio de Janeiro, 1998. 